\title{
The Star-Galaxy Era in Terms of Big History and Universal Evolution
}

Leonid E. Grinin

Eurasian Center for Big History \& System Forecasting,

Oriental Institute, Russian Academy of Sciences

Anton Grinin

International Center for Education and Social and Humanitarian Studies

\begin{abstract}
The present article attempts at combining Big History potential with the potential of Evolutionary Studies. It does not only analyze the history of the Cosmos. It studies similarities between evolutionary laws, principles, and mechanisms at various levels and phases of Big History. Such an approach opens up some new perspectives for our understanding of evolution and Big History, their driving forces, vectors, and trends; it creates a consolidated field for interdisciplinary research. Of special importance is the point that many principles, patterns, regularities, and rules of evolution, which we tend to find relevant only for the biological and social levels of evolution, may be also applied to the cosmic phase of evolution. This is not so surpris-ing, since the formation, life-cycle and renewal of stars, galaxies, as well as other celestial bodies is the longest evolutionary process that took place in the Universe.
\end{abstract}

\section{Keywords}

Star-Galaxy Era, cosmic phase of Big History, laws of evolution, universal evolutionary principles, Universe, preadaptations, Evolutionary Studies, evolutionary selection, additive and substitutive models of evolu-tion, large-scale structures of Universe, gas-dust clouds, non-uniformity concentration of matter, circulation of matter in the Universe, dark and light matter.

Correspondence | Leonid E. Grinin, leonid.grinin@gmail.com

Citation | Grinin, L. (2019) The Star-Galaxy Era in Terms of Big History and Universal Evolution. Journal of Big History, III(4); 99 - 122.

DOI | https://doi.org/10.22339/jbh.v3i4.3444

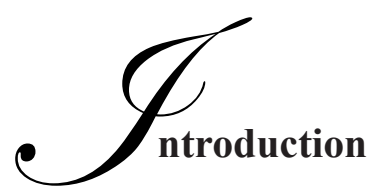

"The effort to understand the Universe is one of the very few things that lifts human life a little above the level of farce, and gives it some of the grace of tragedy." These words said by the Nobel laureate Steven Weinberg (1977: 155) undoubtedly deserve attention, although they dramatize the origins of interest to the problems of the Universe. In fact, we do not need any justification to explain the desire to understand the origins of all living beings. This aspiration to perceive the world in its entirety lies in the human psyche, it is an integral feature of the mind, at least of a certain kind of people who always want to reach out to ultimate causes (although they can never stop on this way). Moreover, in recent decades the investigation of the world in its entirety (but not of its separate aspects) has been intensified in particular within the Big History.

Big History provides unique opportunities to consider the development of the Universe as a single process, to detect vectors of changes of certain important characteristics of the Universe (such as complexity and energy) at various phases of this development. Some authors analyze important general evolutionary mechanisms and patterns, which can be seen at all phases of Big History (e.g. Christian 2004, 2014; Spier 2010; Baker 2013). However, one should note that the Big History studies tend to pay little 
attention to such an important aspect as the unity of principles, laws, and mechanisms of evolution at all its levels. I believe that combining the Big History potential with evolutionary approaches can open wider horizons in this respect (see Grinin et al. 2011). Indeed, common traits in development, functioning, and interaction can be found in apparently quite different processes and phenomena of Big History. In this respect the universality of evolution is expressed in those real similarities that are detected in many manifestations at all its levels.

This article continues author's attempts to combine Big History potential with the potential of Evolutionary Studies in order to achieve the following goals: 1) to apply the historical narrative principle to the description of the star-galaxy era of the cosmic phase of Big History; 2) to analyze both the cosmic history and similarities and differences between evolutionary laws, principles, and mechanisms at various levels and phases of Big History; 3) to show how cosmic evolution fits the universal evolutionary algorithms and correlates with the common evolutionary laws and patterns. Very few researchers have approached this task in a systemic way. It appears especially important to demonstrate that many evolutionary principles, patterns, regularities, and rules, which we tend to find relevant only for higher levels and main lines of evolution, can be also applied to cosmic evolution. Moreover, almost everything that we know about evolution may be detected in the cosmic history, whereas many of the evolutionary characteristics are already manifested here in a rather clear and salient way. One should also bear in mind that the origin of galaxies, stars, and other 'celestial objects' is the lengthiest evolutionary process among all evolutionary processes in the Universe. Such an approach opens new perspectives for our understanding of evolution and Big History, of their driving forces, vectors, and trends, creating a consolidated field for the multidisciplinary research.

While studying the issue it became clear that the number of similarities and common features of the evolutionary progress at its various stages and levels is extremely large, that they can be revealed in the most seemingly different processes and phenomena. Almost everything, that we know about evolution can be already found at its cosmic phase (see also Grinin 2013, 2014a, 2014b, 2015a, 2015b, 2017, 2018, 2019). Naturally, many phenomena are revealed in a rudimentary or unsystematic form, but a number of characteristics, on the contrary, are more clearly expressed just at the cosmic phase. And at the same time, when characteristics and features of biological or social evolution unexpectedly reveal their roots or early forms in earlier phases, it becomes clear that the universality of evolution is a reality found in many manifestations.

Our world is immensely diverse and unlimited in its manifestations. However, fundamentally it is a single world - that is why it is so important to study those fundamentals.

\section{THE FORMATION OF THE LARGE-SCALE STRUCTURE OF THE UNIVERSE}

Preconditions. After the Big Bang, our Universe 'lived' for quite a long period of time without any stars, galaxies, clusters, and superclusters of galaxies (Khvan 2008: 302)1. The formation of modern structure of the Universe lasted for billions of years. However, the first stars and galaxies turn out to have emerged not later than 200-400 million years after the Big Bang (e.g., see European Commission 2011). We are going to discuss this in detail in what follows. And what was the matter from which they had emerged?

Approximately 270,000 years after the Big Bang, a large phase transition occurred resulting in the emergence of matter in the form of atoms of hydrogen and helium. Later, they started to consolidate in new structures (see below). The main mass of this matter concentrated in gas-dust clouds that could have tremendous sizes (dozens parsecs, or even more). ${ }^{2}$

1 See Gorbunov and Rubakov 2012; Guth 1997: Guth A. 2002; Guth 2004; Wood 2018; Grinin 2018 about the Big Bang and the theoretical problems associated with it.

21 parsec $\approx 31$ trillion $\mathrm{km}$. 
Nowadays one can speak about such cosmic fractions as inter-stellar gas and cosmic dust. They can be found either in a very rarefied state or in the form of clouds. But it is known that the clouds we currently observed consist of gas and dust mostly in equal proportions. Therefore, one usually speaks of gas and dust clouds.

For the first time we observe Nature in the role of a constructor. Before that, it had formed just the basic elements. Now one could observe the emergence of enormous structures from tiny particles and 'specks of dust'. After that one could observe this constantly: large-scale structures are composed of myriad minute particles and grains.

The seed grains needed for structuring. The formation of clouds (and later stars and galaxies) meant a concentration of matter on enormous scale, which could have been caused only by gravity. However, this only force is insufficient for structuring, because in 'an absolutely homogenous universe the emergence of large-scale structures (galaxies and their clusters) is impossible' (Dolgov et al. 1998: 12-13). Thus, certain seed grains are necessary - this is comparable with formation of rain drops that emerge around particles of dust or soot; or with formation of a pearl around grit.

Small fluctuations are often needed for the powerful forces to start working. Actually, minor fluctuations (minute deviations from homogeneity) occurred in the Universe early on. Then the larger fluctuations happened. They could act as seed grains for the formation of galaxies and the matter concentrated around them on a much larger scale until the quantity started to transform into a new quality. However, it is not clear what kind of fluctuations caused the formation of galaxies and what is the mechanism of their formation. The initial fluctuations in other evolutionary spheres also often remain a mystery.

This is a perfect example of the point that the nonuniformity (in particular with respect to the distribution of matter, energy, etc.) is a universal characteristic. Any major evolutionary shift in biological and social systems is preceded by the concentration of certain forms, resources and conditions in certain niches and places. The higher the stage of evolution is the more significant this process is. Thus, in the major system the common processes may proceed in their usual way, whereas in the concentration zone some peculiar processes start (this is what takes place in star formation zones).

Thus, we might formulate an evolutionary rule of important heterogeneity and fluctuations. The emergence of critical heterogeneity which can be the core of changes is often required for the evolutionary change (even within the frameworks of a typical transformation without qualitative evolutionary growth). An absolute homogeneity makes some evolutionary processes impossible while even a slightest difference can trigger the regrouping of matter or elements in the assemblage. And a new structure and order arise on this basis (see Grinin 2017, 2018 for more details).

The epoch of formation of the large-scale structures in the Universe. The first galaxies and stars

Dark and light matter. Nowadays it is generally accepted that dark matter plays an important role in the formation of the first galaxies, as it appeared capable of consolidating into clusters much earlier than the light (baryon) matter. The latter could not contract until the end of the hydrogen recombination (atom formation) due to radiation $(270,000$ years after the Big Bang). Only when hydrogen nuclei and electrons were able to merge and form atoms, whereas photons separated from the matter and flew away, the pressure of the radiation dramatically dropped. Perhaps, we observe here a very interesting evolutionary pattern of a transition from disorder to order and decreasing entropy. This case correlates rather well to Herbert Spencer's universal principle of evolution (Spencer 1862: 495, 1970: 396) $)^{3}$. He formulated it as a law

3 It is interesting that he gives an example connected with the Solar system. "If the Solar System once existed in a state of indefinite, incoherent homogeneity, and has progressed to its present state of definite, coherent heterogeneity; then the Motion, 
of the transition of a substance during its qualitative development from indefinite (non-differentiated and non-specialized) homogeneity to definite (more specialized) heterogeneity. As a result, the light matter would fall in potential holes prepared for it by the dark matter. Though the dark matter was initially more capable to structuring than the light matter, the progress toward structuring turned out to be very short and leading to almost a dead-lock. ${ }^{4}$ Meanwhile, the evolutionary potential of the light matter was based on the 'achievements of the dark matter'. Such a model of development is rather typical for evolution. For example, long before the transition to agriculture some gatherers of cereal plants invented many things (sickles, granaries, and grinding stones) that later turned to be rather useful for agriculturalists, whereas specialized hunter-gatherers turned out to be an evolutionary dead end.

The epoch of formation of the large-scale structure of the Universe. First galaxies and stars. There are rather diverse opinions on timing, process characteristics and sequence of formation of stars, galaxies, galaxy clusters and superclusters. There is a hypothesis that galaxy protoclusters were first to originate. According to it the same process that engendered galaxy could operate on a larger scale, and the first generation of gas clouds being protoclusters, that fragmented to form galaxies (Peebles 1980). Similar phenomena can be found at higher levels of evolution, when something general is formed (which will later turn into a larger taxon in the hierarchy), that later differentiates into lowest-order taxa. That is how species and classes are formed in biology. The same refers to a society: at first there emerge rather large formations, like families of languages and then

Heat, and Light now exhibited by its members, are interpretable as the correlatives of pre-existing forces; and between them and their antecedents we may discern relations that are not only qualitative, but also rudely quantitative.

4 However, as with any evolutionary dead end, this does not mean an absolute stagnation. At present, in galaxy halos the dark matter is structured in certain smaller structures (see, e.g., Diemand et al. 2008). the languages, ethnic super-groups and then ethnic groups, and sometimes early large empires or states; and afterwards within their framework statehood goes one or two levels down. In other words, there emerges a non-differentiated large structure which is capable to produce a great number of peculiar structures.

However, a more commonly held hypothesis suggests that protogalaxies (in the form of giant condensed gas clouds) were the first to emerge within the structure of the Universe, and later they became the birthplace for separate stars and other structural elements (see, e.g., Gorbunov and Rubakov 2011).

However, in recent years new evidence has come to hand to support the idea that those were the stars that appeared first. This discovery somehow modified the previous theories. At present, it is widely accepted that the stars were first to emerge, but those were the giant stars, much more massive than most of the later-formed ones (May et al. 2008). Because of the absence of carbon, oxygen and other elements that absorb the energy from condensing clouds, the process proceeded more slowly in that epoch; thus, only giant clouds could condense producing massive stars hundreds times larger than the Sun (Ibid.). Nowadays we can also find such giants which have 100-200 solar masses, but they are considered to be unstable (see Surdin, Lamzin 1992). Such giant stars lived only a few million years (the larger is star, the shorter is its life). In addition, the first stars contained a small amount of heavy elements. Thus, more than one generation of stars could change, until the quantity of heavy elements gradually increased. The emergence of 'heavy elements' from the 'dead star stellar remnants' resembles the formation of marine sediments or fertile soil at the Earth's surface from the accretion of skeletons or conversion of remnants of dead plants. The circulation of matter in the Universe is always observed everywhere and at all levels.

In recent years we have witnessed the discovery of a few galaxies that are claimed to be the oldest in the Universe. Meanwhile, the dates of formation of the first galaxies are shifted closer and closer to the Big 
Bang. The emergence of the first galaxies is dated to less than 400 million years after the Big Bang; and there are even claims that some more ancient galaxies have been discovered. They are claimed to have emerged only 200 million years after the Big Bang (see European Commission 2011). The evidence on the first stars refers to $c$. 150-200 million years after the Big Bang - hence, stars and galaxies appear to have emerged almost simultaneously. Since that time the substance in the Universe coexists in three major forms, depending on its density: in a dense state in celestial bodies, in a rarefied form in clouds of different sizes, and in a highly rarefied state (dozens of times if compared to clouds) in interstellar gas.

Thus, galaxies were actively formed during the first 300-400 million years after the Big Bang. However, although some structures emerge already at that time, still they were not yet dominant in the Universe, and they were still very amorphous and crumbly (Hawking 1998). The formation of a more or less stable largescale structure took some time, probably a couple of billion years. As we mentioned above, the formation of a number of heavy elements without which the creation of resilient stars was impossible also took time. The formation of the Universe close to the modern structure could hardly take place quickly.

\section{THE ERA OF THE STAR-GALAXY STRUCTURE OF THE UNIVERSE}

The whole history of the star-galaxy phase of cosmic evolution is basically the history of formation of various structures of different size, as well as their merging into larger structures (but it is a history of their disintegration as well). The formation of galaxies and their clusters, as well as of stars and other celestial bodies was the longest evolutionary process that had ever taken place in the Universe. At present we observe that this process is still going on alongside changes and disappearance of galaxies and stars. During the first eight billions years, the formation of huge diversity of stellar bodies and new heavy elements took place in the Universe until about 5-4.5 billion years ago there the conditions were formed for the formation of stellar
(Solar) system. On one of its planets there started new geological, chemical and biochemical processes (for more detail see Christian 2004; Lin 2008; Batygin et al. 2016; Grinin 2017, 2018).

\section{The structure of the Universe}

\subsection{Evolutionary principles of the structure of} the Universe. The formation of galaxies and their clusters was probably the process that lasted for billions of years. Some principles describing the basic structure of the Universe may be applied to different levels of evolution (below we will consider just two of them).

1) The combination of antagonistic qualities. For example, in the structure of the Universe one can find the combination of uniformity and non-uniformity. The uniformity is already manifested at the inflation phase, when the Universe started inflating evenly in all dimensions. The uniformity has preserved till present, but only at the largest scale (of an order of magnitude of 100 megaparsecs $^{3}$ ). For reference, the size of the largest galaxy clusters ( such as our Local Group with the center in the Virgo constellation) is 40 megaparsecs at most (Gorbunov and Rubakov 2011). The non-uniformity of the Universe is manifested at scales smaller than 100 megaparsecs; and the smaller is the scale, the more salient is the unevenness. The combination of antagonistic qualities is a phenomenon that is rather characteristic for many other evolutionary levels. Thus, the antagonistic notions like 'even surface' and 'uneven surface' are quite applicable to the Earth surface: at bird's eye it looks even.

2) Density and sparsity can be traced everywhere, starting from the atomic structure, where the mass is concentrated in a tiny nucleus, while most of the atom is an empty space. There is a huge non-uniformity between the scale of the Universe and the space that the main mass of the light matter occupies within it (at least, baryonic) matter. It is concentrated, first of all, in stars which actually occupy only a $10^{-25}$ part of the total volume of the Universe (not taking into account the galaxy nuclei [Pavlov 2011: 43]). Were there such proportions in the ancient Universe? Probably, 
not. Therefore, the concentration of the matter is increasing. Not only the hard matter is distributed very unevenly throughout the Universe; the same is true of the gas. Much of this gas is concentrated in giant molecular clouds which are of many thousands of solar masses (Lipunov 2008: 37).

At the same time the difference in density is fractal, which is especially evident in the high-density zones. The factors contributing to such unevenness are not always clear; for example, it is not clear, what the uneven distribution of masses during the formation of galaxies (Weinberg 1977) as well as many other processes of distribution, concentration and dissipation are connected with.

But the principles of uneven distribution of the matter mass at different evolutionary levels are rather similar. For example, at present the main mass of the Earth's population is concentrated in a rather small territory in comparison with the total territory where life on the Earth is possible.

\subsection{The structure of the contemporary Universe}

The main structural elements of the Universe are galaxies, their clusters, and superclusters. Superclusters basically form our Metagalaxy ${ }^{5}$. All the structural elements are rather stable in terms of gravitation, though they can split, merge, and collide.

Galaxies are integral structural entities with a rather complex structure which includes, in addition to regions and arms, a nucleus (core), semi-periphery (so called 'disc'), and periphery (so called 'halo') (Baade 2002: 255). The halo consists of both single stars and various stellar clusters. The halo's radius (a few hundred thousand light years) is much larger than the radius of the galaxy's disc. ${ }^{6}$

5 If, according to some researchers, the Metagalaxy is not the only one in the Universe, then for some time it will be considered as the largest structural unit of the Universe (see Pavlov 2011: 52). If the Universe is not a Universum but a so-called Multiverse, then the Universes or their groups will be the maximum unit of the structure (see Vilenkin 2006; 2010).

6 There might be an invisible halo consisting of dark matter behind the visible halo. It may be found in many (if not all) galaxies, whereby the diameter of the dark halo might exceed
According to Hubble, the galaxies are classified into spiral, elliptical, and irregular with various subtypes (Ibid.: 18-32); yet, by now one more galaxy type has been identified - the lenticular galaxies.

A galaxy contains around 100 to 200 billion stars. There are small (dwarf) galaxies with a few million stars, there are also giant galaxies consisting of up to a trillion stars. Our galaxy with its mass of about $10^{11}$ solar masses is one of the largest ones. It contains 200-300 or even more billions of stars. However, the mass of our neighbor - the Great Andromeda Nebula (M31, found in our cluster of galaxies (the Local Group) - is about three times larger. Probably, the most famous M87, which is situated in the central part of the cluster of galaxies in Virgo constellation, has the largest mass. Apparently, the mass of this Galaxy exceeds by hundreds times the mass of our Galaxy. At the other pole there are dwarf galaxies whose masses are $10^{7}$ of solar mass, that is several dozens of times more than the mass of globular cluster (Shklovskii 1978: Part 1, Chapter 6).

Stars are distributed rather unevenly throughout galaxies, stars are parts of various groups and clusters; some of them consist of just a few stars, but some clusters can contain a few million stars. For example, within our Galaxy more than 1,500 star clusters have been identified (Surdin 2001). There are many globular clusters - spherical clusters tightly bound by gravity and consisting of hundreds of thousands, as a rule, rather old stars. These are old stars (there are 150-200 in our galaxy). There are about 22 per cent among our nearest galaxies. In these galaxies, a bright main flattened body, 'a lens', is surrounded by a weak halo. Sometimes the lens is surrounded by the ring (see: Novikov 1979: ch. 1, § 8). More than half of the members of the Local Group are the older elliptical galaxies with intermediate or minor

the diameter of the visible halo by an order of magnitude (see Ryabov et al. 2008: 1131). 
luminosity (Novikov 1979: 31). Galaxies are complex and (to a considerable extent) self-regulating systems, within which some stars disintegrate, whereas new stars form from cosmic gas and dust. The circulation (which results in processes of renovation of matter and its mixing) takes place at all levels of the Universe - both spatially and at different levels of evolutional complexity.

An average galaxy cluster consists of $500-1000$ galaxies. Galaxy clusters have a rather regular structure which is likely to include a massive nucleus in the center. Galaxy superclusters are entities consisting of 2-20 galaxy clusters and galaxy groups as well as of isolated galaxies. In general, there are known more than 20 superclusters, including our Local Group (Gorbunov, Rubakov 2012: 4).

\subsection{Generations of galaxies and stars}

There are rather diverse opinions on the number of generations throughout the evolution of the Universe. In addition, there is no consensus on which galaxies should be regarded as old, and which galaxies should be considered young. The point is that within a single galaxy one can find stars and their aggregates that considerably differ in their type, age, and other parameters. For example, the age of our Milky Way galaxy is more than 12 billion years, but that is the age of just its halo while many stars in its branches are only two-five billion years or less. Yet, it appears possible to single out a few widely accepted basic ideas.

1) In the evolution of the Universe, there have been three (or at least two) generations of galaxies and stars. In general, old galaxies are smaller and dimmer. Their stars contain dozens of times smaller quantities of heavy elements than the Sun. The astronomers can hardly observe any star formation processes within such galaxies. There is also a hypothesis that more dark mass is concentrated in old galaxies in comparison with younger ones. The same way, older and younger stars differ from each other in their size, luminosity, and chemical composition.

2) It is difficult to speak about a clear periodization of generations of galaxies, because of the ongoing process of formation of galaxies and stars. Galaxies need to constantly renew their composition in order to retain their identity. As Iosif Shklovskii maintains, in this respect galaxies are very similar to primary forests with its mix of tree ages (whereas the age of trees is much less than the age of the forest itself [Shklovskii 1978]). The motility and variability of the celestial landscape resembles very much the motility of geological landscapes.

3) The formation of galaxies can proceed in different ways, for example, through the absorption of smaller galaxies by the larger ones in particular as a result of a collision. If a small galaxy comes into collision with a massive one, it is absorbed by the latter and loses its identity. Every time when passing near a massive galaxy the stars estrange from it (May et al. 2008). In this case both young and old clusters and groupings of stars are combined (see about it below). Another way is merging. Galaxies of younger generations can sometimes form through the accretion of a few small, weak and compact galaxies into a single galaxy. In this case they became 'building blocks' for galaxies. Finally, it may happen that two large galaxies collide. Such a collision may take billions of years and be accompanied with active star formation and emergence of very large and bright stars. The latter means that these are short-living stars, that is there will be many bursts of nova and supernova. Finally, galaxies may diverge again, but in this case they turn out to be very different from what they used to be before the collision, whereas one more galaxy may emerge out of the matter estranged from the both galaxies (see May et al. 2008: 142).

There are numerous analogies to those models of galaxy formation in biological, geological, and, especially, social evolution. As stars and galaxies are composed of more or less homogenous matter (that can be divided or united rather easily), they somehow paradoxically resemble societies that consist of people who can be included into other societies through integration or capture. On the other hand, captures are 
also attested among social animals (e.g., among ants see Genet 2007).

4) Galaxies are collections of different types of stars. However, there are certain peculiarities as regards the position of old and young stars within galaxies which is probably connected with the self-regulation within the galactic systems or the peculiarities of star formation which occurs in large groups, or due to other factors (about self-regulation in cosmic world see Grinin A. 2016). Thus, within our galaxy the younger stars (such as the Sun which is a few billion years old) are generally larger, hotter and brighter. They are located toward the disc plane, and, especially, within the galaxy arms; whereas in the galaxy periphery (in its halo) one would find older stars more than 12 billion years old (which suggests the overall age of our galaxy). Yet, older and younger stars may be also located rather close to each other. Thus, one may find many old stars near the galaxy center (bulge), but there are also young stars that emerged from the matter produced by the disintegration of older stars. The highest stellar density is found in the galaxy center where it reaches a few stars per cubic parsec.

As we already mentioned, the very old, not bright, not hot and not massive stars contain many times less amounts of heavy elements than the Sun. It is not surprising that these stars and young, hot, and bright stars of the surface of disc, arms and halo were labeled differently as 'Population I' and 'Population II' respectively. ${ }^{7}$ It is generally accepted that the majority of globular clusters are very old (12 billion years old or even more). But the dispersed or open galactic clusters are dozens or even hundred times younger than globular clusters (i.e. they are just hundreds of millions of years). But there are younger stellar associations (see, e.g., Surdin and Lamzin 1992; Surdin 2001).

On the one hand, the preservation of generations of

7 Note, that when there appeared an evidence of the existence of the stars of the first generation which emerged at the age of the Universe of 150 million years from the moment of the Big Bang (see about it above), they were named 'Population III' in order to follow the conventional designation. stars and galaxies demonstrates an additive character of the evolution of abiotic systems, whereas we can see elements of substitutive model of evolution at biological phase and its full system at social phase of Big History. However, the capture of stars and galaxies with their subsequent integration and prolonged processes of collision of galaxies demonstrates that in abiotic natural systems one may still find some other models of evolution - connected with 'wars' and 'submission of outsiders'.

The type of development through the emergence of different generations of individuals and species (preserving certain genetic features, on the one hand, and accumulating important changes in their structure and characteristics, on the other) is rather widespread at all phases and levels of universal evolution. Within any biological class or order (e.g., perissodactyls) we can see how important characteristics vary and gradually change from one species to another, whereas due to those characteristics some species press out others and occupy better niches (see, e.g., Grinin, Markov, and Korotayev 2008). Various types of states and civilizations also rather vividly illustrate the progress: for example, more organized and developed states emerge through the absorption of the achievements of less developed generations of states, which one can illustrate using examples from the history of Ancient Rome, Byzantium, some Medieval European states and so on. The coexistence of different generations sometimes leads to the situation when younger and more advanced entities either transform the older ones or form a symbiosis with them (though in some places one may find 'restrictions' for older types and generations).

\subsection{Change of the chemical composition of the Universe}

Hydrogen has always been the most abundant element in the Universe chemical composition; yet, its share constantly decreased. This occurred (and occurs) because hydrogen is the main fuel for the nuclear fusion reactions that support life and luminosity of stars. Despite the tremendous amounts of energy 
released during these reactions, the energy release rate is very low. For example, the intensity of the solar radiation is $2 \mathrm{erg} / \mathrm{g} \cdot \mathrm{s}$, which is almost equal with a pile of burning leaves. Stars shine brightly because they are massive and large (Surkova 2005: 9).

Increasing temperatures inside the core of some stars were needed for the formation of new elements that were absent in the era of recombination. However, all of the fusion reactions that occur to produce elements larger than iron no longer release energy. Reactions of another type are needed for the formation of elements heavier than iron - those reactions consume more energy than release. That is why there are such relatively small amounts of heavy elements in the Universe. Yet, such peculiar reactions do take place for example, in neutron stars and during explosions of supernovas. In supernovas, during their bursts, within about just 100 seconds heavy elements from the end of Mendeleev's periodic table are formed, including uranium and thorium (Surkova 2005: 9).

When supernovae explode, heavy elements are expelled through the Universe with stellar winds and through the fall of the dispersed matter on the surface of cosmic bodies (so-called accretion). As stars turn to be the main centers of the synthesis of chemical elements, the distribution of heavy elements in the Universe is very inhomogeneous.

The emergence of heavy elements and their concentration in certain bodies and compositions are extremely important processes, which lead to an enormous increase in the number of matter combinations, and consequently have an evolutionary potential; in particular, they lead to the start of the fullscale chemical, biochemical, and biological processes. In certain respects, such a slow and uneven accumulation of new structural elements (heavy elements) resembles the process of an accumulation of valuable mutations in biological evolution, or the accumulation of valuable innovations in social evolution (all of them bring the expansion of the evolutionary potential and increase the rates of evolutionary changes).

Thesimilarities and differences of stellarcompositions (the presence of heavy elements) somewhere resemble similarities and differences in genome. All living organisms mostly have its similar structure and all huge differences are caused by small divergences (by several percent) in genes.

\section{The Evolution of Galaxies and Stars}

\subsection{Processes of the formation of galaxies and stars}

Until quite recently, the processes of star formation were entirely concealed from an external observer; however, at present due to the technological progress one can observe some aspects of those processes in many parts of our galaxy. Those observations confirm the theory of stellar formation from cold clusters that are heated by gravitation and pressure.

Briefly, this process may be described as follows. Within giant hydrogen and helium clouds, some heterogeneities emerge, which launch (under certain conditions) the gravitation processes that start to collect that mass into spherical forms. Sometimes a direct formation of a giant mass of gas clouds takes place, from which a galaxy or a star cluster later emerges. In this case the cloud fragmentation may occur and thus, more and more gas-cloud spheres (there could be hundreds of millions, or even hundreds of billions of them) emerge, which can gradually transform into protostars. This process continues up to the point when the gas density becomes so high that each new fragment already has a mass of a star (Surkova 2005: 49). Then the gravity starts impeding further fragmentation. This process is denoted as 'cascade fragmentation'. It is remarkable that it resembles certain processes in social evolution - for example, the fragmentation of large early states into separate parts that decentralize up to the point when further division becomes unreasonable (e.g., in certain periods there were dozens and hundreds of independent states in the territories of Germany or France).

As enormous gas/dust clouds appear unstable, they disintegrate into large bundles, so the formation of stars proceeds in groups. This phenomenon is of interest not only with respect to stellar evolution. The group 
formation is rather typical for evolution in general (in this way populations and sometimes new species emerge; chiefdoms, city-states, and sometimes political parties emerge in groups, and so on).

The further process of the star formation is connected with the point that the initial compression heated the gas to a rather high temperature that, on the one hand, prevents the further compression of the gas, and, on the other hand, eventually contributes to the onset of the nuclear fusion reaction (Hawking 1998).

\subsection{Diversity of stars and galaxies}

Diversity is an absolutely required condition of evolutionary development. And this condition is fully realized within cosmic evolution. As has been mentioned above, galaxies differ in their types, age, size, and structure. They also differ in many other characteristics including the chemical composition and the immediate system to which they belong, for example whether they are binary or isolated stars, whether the stars have a planetary system or not, etc. Those differences may vary greatly.

The varying impact of gravitation and resulting peculiar behavior of gas-cloud masses could become the reason of formation of different types of galaxies. That means that galaxies are born either as spiral or elliptic ones and the type of galaxy is preserved in the course of its evolution. In particular, a galactic structure is to a large degree determined by the initial conditions of its formation (e.g., by the character of rotation of the original gas clump from which a galaxy is formed).

Stars differ in mass, temperature, chemical composition, luminosity, age, and other characteristics. Those differences may vary greatly. For example, with respect to masses, stars range in mass from about 0.1 to 100 or more solar masses. There are some considerations that the thermonuclear fusions cannot go in a star with the mass of less than $8 \%$ of the Solar mass that is why such objects are not attributed to stars. It is rather natural that the number of smaller entities is orders of magnitude larger; ${ }^{8}$ actually, the

8 Thus, for every ten million red dwarfs we find only 1,000 same phenomenon may be observed, for example, in Zoology or Political Geography where the number of small animals or countries is much larger than that of large ones.

\subsection{The life-cycle of a star: Stages of stellar birth, aging, and death}

Protostars. As mentioned above, stars emerge through the condensation and compression of gas clouds under the influence of gravitational forces. This is a protostar phase. In comparison with the subsequent life of a star, the period of its slow contraction seems rather short; however, actually this is not a quick process as it continues sometimes up to 50 million years (Surkova 2005: 50). During this period of time, there is a tremendous rise in the temperature at the core of the protostar, the temperature may grow up to 8-10 million Kelvin, and, as a result, thermonuclear reactions become possible. The protostar becomes a young star. However, an external observer will only be able to see it in a few hundred thousand (or even a few million) years when the cocoon of gas and dust surrounding the protostar dissipates.

Actually, we deal with a sort of miracle - a giant shining incandescent body, which is capable of living for billions of years, emerges from an absolutely amorphous, lacking any structure, opaque, and cold mass of gas mist. In other words, we deal here with a vivid example of self-organization that takes place under the influence of gravitation and thermodynamic laws. In particular, an intensive contraction leads to heating, which increases the internal pressure, which, eventually, stops the compression process (for more details about the processes of young stars emergence including the direct observation of them as well as about the Sun's birth see Marakushev et al. 2013; Marov et al. 2013; Grinin 2017, 2018).

One may also note that the emergence of stars and galaxies must have a certain trigger that creates turbulence and heterogeneity. Those triggers and catalyzers are the inherent components of evolutionary

giants and one supergiant (Surkova 2005: 26). 
mechanisms that may be found in many processes: in chemical and geological processes, within biological evolution with respect to fast formation of species, or within social evolution with respect to state formation (see Grinin 2011 for more details). The supernova shock wave, the collision of a molecular cloud with spiral arms of a galaxy and other events can become such a trigger of the star formation (Surkova 2005: 50).

Another (the longest) macrophase is the main sequence star. During this phase of the stellar lifetime, nuclear-fusion reactions that burn hydrogen to helium in the core, keep the star shining. That is why the duration of the main sequence phase depends mainly on the stellar mass. The more massive is the star, the shorter its lifespan on the main sequence (as with a larger mass the 'fuel combustion' processes run more intensively). A star preserves its size and form due to the mutual struggle of two forces: the gravity that tries to compress the star and the gas pressure produced as a result of nuclear reactions and powerful heating. There is a dynamic equilibrium between temperature and gas pressure. With growing temperature, the gas expands and works against the gravitation forces, which results in cooling of the star; this way the thermal balance is kept. In the lifetime of stars and galaxies, as well as at all other levels of evolution, we find numerous cases and different forms of the interaction between two opposite processes which make it possible for 'individuals' to live. The processes of assimilation and dissimilation support vital activities within biological organisms; the processes of animal reproduction and their extermination by predators support the population balance; interaction between processes of production and consumption is the basis of the reproduction of social systems, and so on.

Red giants. The new phase of stellar evolution is connected with the exhaustion of hydrogen supplies. The gas pressure (that maintained the star balance when necessary fuel was available) decreases and the stellar core compresses. This leads to a new increase in temperature. A star starts to burn heavier elements and thus, the stellar composition significantly changes. Simultaneously with the compression of the core, the star's outer layers expand (they can even detach to form a gaseous nebula). In general, the star inflates and expands a few hundred times, and it transforms into a red giant, and then with further expansion into a red supergiant (large stars with more than ten solar masses transform directly into supergiants). This phase lasts for about one tenth of the 'active lifetime' of a star, when the processes of nuclear fusion go on in its depths.

Star death: three cases. The next phase is the transformation of a red giant or supergiant. Actually, the new form depends on stellar mass and a number of other characteristics such as the stellar rotation and velocity, the degree of its magnetization, and so on. The following three outcomes are considered most typical. They depend on stellar mass (but the limit value estimates vary significantly, and so below I will mention the main alternative values after the slash). ${ }^{9}$ Stars with the masses smaller than $1.2-1.4 / 3$ solar masses transform from red giants into the socalled 'white dwarfs', when the star sheds its outer envelope to form a planetary nebula with an extremely contracted core (down to the size of the Earth). The further compression does not occur because of the socalled degenerate electron gas pressure that does not depend on temperature. As a result, the white dwarf is rather stable. However, due to the lack of hydrogen and helium, thermonuclear fusions can no longer proceed within such a star. A white dwarf is very hot when it is formed; yet, afterwards the star cools and transforms into a 'black dwarf', that is, it becomes a cold dead cosmic body.

For stars with an initial mass of more than 1.2-1.4, but less than 2.4-3 (in another calculations from 3 to 7-10) solar masses, their slow and gradual aging results

9 According to one of classifications (that might be more correct than the one reproduced below), it appears possible to subdivide all the stars just in two classes: a) massive stars (with a mass exceeding $c .10$ solar masses), producing neutron stars and black holes, and b) non-massive ones producing white dwarfs (Lipunov 2008: 99). 
in an 'infarct', that is a collapse. After the depletion of hydrogen and the decrease of the internal gas pressure (that used to balance the gravity), under the influence of gravity the core gets extremely compressed (by dozens thousand times - up to the radius of ten kilometers) just in less than a second. Almost simultaneously the external layers of the star are blown away with a huge speed as a result of shock wave. This supernova shines brighter than millions of ordinary stars, but for a very short period of time. This explosion expels the stellar material into interstellar medium and thus, there occurs the formation of considerable quantities of heavy (heavier than iron) elements that afterwards concentrate in various celestial bodies. The remaining core contracts to become a neutron star (which is supposed to contain super dense neutron fluid) In its size, such a star is 5 billion times smaller than the Sun, but it is hundreds of thousands of times brighter because the temperature on its surface is 1000-1500 times higher than on the Sun (Lipunov 2008: 133).

If stellar mass exceeds the limit of 3/7-10 solar masses, after hydrogen is burnt out it will start collapsing and explode (though sometimes it may collapse without an explosion), but the force of compression will be unlimited, as the gravity becomes enormous because of the huge mass and absence of internal forces that can prevent the collapse. The action of the gravitational force which is balanced by nothing leads to the situation when the stellar diameter becomes infinitesimally small. According to theoretical calculations, the star is transformed into a black hole whose gravity fields are strong for light to escape.

\section{UNIVERSAL EVOLUTIONARY PRINCI- PLES \\ IN THE STAR-GALAXY ERA}

\section{Life, Death, and Catastrophes in the Evolution- ary Aspect}

The irreversible character of evolution is its most important characteristic. It can be observed as a steady movement to more complex structures and forms of organization, to changing chemical composition of the Universe, etc. As regards the individual objects, the irreversible character of evolution is obvious and undoubted. A star which passed through a certain phase of life cannot reenter this phase.

The stellar lifetime in terms of maintaining and breaking the dynamic equilibrium. At the initial phase a cloud of gas 'burns' itself under the compression like packed straw or rags ignite spontaneously. The next phase of self-organization is connected with the formation of complex stellar structure on the main sequence phase during which burning out of hydrogen occurs. After burning out of the most part of hydrogen a star enters a new phase, it expands and transforms into a red giant. At the same time the processes of self-organization start again and the stellar structure radically changes (highly compressed core coexists with the expanded envelopes). After the fuel is burnt out in a red giant, the next phase is compression under the influence of the gravitational force and formation of a brand-new structure: small but very massive core with extremely high density of the matter within it.

Let us consider the stellar life in terms of maintaining and breaking the equilibrium. First of all, there is a thermal equilibrium, when the rate of energy produced in the core (through thermonuclear fusions) balances the loss of energy through the emission of radiation into space. This equilibrium is broken when hydrogen fuel is gone. The reserves are apparently compensated when a star starts using another type of energy. This may occur through the contraction of the star which begins fusing helium into carbon, thus producing many times more energy for every atom; afterwards heavier elements may be used as fuel, and each heavier element will produce more and more energy per atom. Meanwhile, the core of the star begins to increase in temperature. There is equilibrium in terms of pressure of different forces and preservation of a certain form and size of the star. Within the main sequence phase, the balance is maintained as the gravity pulls all the stellar matter inward, toward the core, while gas pressure pushes heat and light away from the center. 
This pressure exists until the reserves of nuclear fuel are exhausted (Efremov 2003: 97). With respect to red giants one may speak about equilibrium of another kind in two dimensions. In the core the temperature grows due to contraction and thermonuclear reactions of higher levels (described above) start; as a result of those reactions the temperature may grow up to 100 million Kelvin. That is why a stronger gravity is balanced by a stronger (due to temperature) gas pressure. In the meantime, within the shell the equilibrium is achieved through the multifold expansion of the outer layers. In neutron stars and white dwarfs, the subsequent phases of the stellar lifetime, there is their peculiar equilibrium.

The problem of the individual's death. Death as an opportunity for life to go on. Stellar life and death can hardly leave anybody indifferent. Actually, within the Big History framework, this is the first time when we come across the problem of a life cycle of individual objects in such an explicitly expressed form. On the one hand, the star's fate, lifespan, and type of death depend on initial parameters, as if they were 'genetically programmed' (and, hence, they may be forecasted); on the other hand, they may be altered by some contingencies. Thus, the star's fate is not 'fatal', indeed. Binary star systems increase highly the variability of the individual star fates; as Lipunov (2008: 252) puts it, we deal here with a kind of 'quadratic evolution'. What is more, it is actually possible to speak about differences in the 'individual' stellar behavior or 'within a group', because the interaction of two, three, and more stars may lead to very significant differences and unusual results that cannot emerge within the development trajectory of individual stars. In fact, similar patterns are observed at other levels of evolution, when behavior of pairs or groups of individuals produces outcomes radically different from the ones observed with respect to the behavior of an individual not interacting with others.

Finally, the meaning of individual's death for evolution may be different. Up to a certain degree one may observe a direct correlation between the 'strength' of death, the power of the stellar explosion, and the formation of conditions for a new evolutionary search. Stellar explosions affect the dynamics of their environment; consequently, they may help create unusual conditions that contribute to the emergence of certain developmental deviations. Within tens of thousands years the zone of explosion expands to a vast area of interstellar medium (covering the distances of dozens of parsecs); in this area one can see the formation of new physical conditions (in particular, temperature, density of cosmic rays and magnetic fields strength). Such a disturbance enriches the respective zone with cosmic rays and brings changes to chemical composition (Shklovskii 1978). The explosions also contribute to star formation. Thus, a star does not die in vain. One can draw here an interesting analogy with extinctions in biological evolution which contribute to new directions of speciation. The stellar destruction can be also compared with the disintegration of large empires with all the subsequent repercussions. The disintegration of a large empire leads to a cascade of new states forming both in the place of the empire and even beyond its borders. Historical detonation contributes to politogenesis the same way as the cosmic detonation contributes to star formation.

Structuring, self-organization and nesting-doll structure The whole history of star-galaxy phase of cosmic evolution is the story of formation of various structures with different size and grouping of these structures into larger ones. At the same time, as we already mentioned, we deal here with the ability of objects to self-organize at all phases of universal and individual evolution. It is very important that structuring occurs not only among stars and galaxies but also among molecular clouds. The latter can be regarded as a parallel branch of evolution. Parallelism plays a great role in evolution dramatically increasing the opportunities of transition to something new and creating a field of contacts between various directions of evolution (see about it below).

They generally have a rather complex 'Russian nesting doll' structure, whereby smaller and denser 
condensations are placed within larger and sparser ones (see Surkova 2005: 48). The Russian-doll structure is also typical for higher levels of evolution. Thus, smaller groups of social and gregarious animals constitute larger groups and tend to reproduce their structure. The same refers to social evolution, in particular to the non-centralized entities: for example, the tribal formations, whose constituent parts (lineages, clans, and sub-tribes) often reproduce the structure (and structural principles) of the tribe. That is why tribes can easily split and merge when necessary. The same is true of herds of gregarious animals.

Synthesis of gradualism and catastrophism. With respect to cosmic evolution one may observe a combination of two principles that provoke endless discussions in geology and biology. The subject of those discussions is what principle prevails in evolution. Are we dealing mostly with slow gradual changes, eventually leading to major changes (gradualism)? Or, does the development mostly proceed through sharp revolutionary breakthroughs which in biology are often connected with catastrophes? Within star-galaxy evolution the combination of both principles is more than just evident. Here, as at no other evolutionary level, both modes of evolution are organically combined in individual fates of the stars. The main sequence phase of stellar evolution (when the fusing of hydrogen occurs) demonstrates the gradual character and the importance of slow and prolonged processes. However, catastrophes of various scales can take place within the lifetime of any star. For some stars, such radical changes may manifest in major - but still local - changes (such as shedding the outer layers), whereas for other stars these might be tremendous catastrophes when stars die, figuratively speaking, 'brightly' and 'heroically', illuminating the Universe, leaving a billion-year-long footprint of light. The latter, that is the extraordinary phenomena and events, both among the stars and among humans are less numerous than the former, that is the common ones.

\section{Some Evolutionary Ideas in Connection with the Star-Galaxy Phase of Evolution of the Universe} In the evolutionary process of formation of stars, gal- axies, nebulae, and cosmic clouds one can distinguish a number of important evolutionary principles and laws that are not evident. Their detection is important for understanding the unity of principles of development of the Universe. Those principles and observations are grouped below into several blocks.

\subsection{Evolution proceeds with constant creation and destruction of objects}

Nature, when creating, destroying, and renewing various objects, 'tests' many versions, some of which turn out to be more effective and have more chances to succeed in terms of evolution. For such a situation of selection within constant destruction and creation process, it appears possible to apply a rather appropriate notion of creative destruction introduced by Josef Schumpeter (1994).

- 'Evolution is stronger than individual objects'. Cosmic processes are accompanied by constant emergence, development, change, and death of various objects (stars, galaxies, and so on). Thus, here one can point as relevant the principle that was expressed by Pierre Teilhard de Chardin (1987) with respect to life in the following way: 'life is stronger than organisms', that is, life goes on exactly because organisms are mortal. The same is relevant to stellar evolution. We may say here that the cosmos is stronger than stars and galaxies; and in general, evolution is stronger than individual objects.

- Rotation and keeping balance take place due to constant destruction (or transition to new phases in the lifecycle) of some objects and the emergence of others. This keeps balance and creates conditions for development, because development is a result of change of generations and species.

- In every end there is a beginning. Starevolutionary 'relay race'. The material of dead objects becomes building blocks for the formation of new objects. This represents the circulation of matter and energy in nature; on the other hand, this represents a sort of 'relay race'. ${ }^{10}$

10 For more details on the 'rule of evolutionary relay race' 
The latter allows using the results of long-lasting processes (in particular, the accumulation of heavy elements). ${ }^{11}$ Thus, we deal here with the above mentioned 'creative destruction' - the creation of new objects due to the destruction of the old ones, which ensures continuity and provides new forms with space for advancement (e.g., the change of generations of biological organisms always results in certain transformations). The change of rulers may not necessarily lead to radical social changes; however, each new ruler is somehow different from his predecessor, as a result the accumulation of historical experience occurs.

- New generations of organisms and taxa are a mode of qualitative development. One may also detect generations of taxa, which already have significant evolutionary and systemic differences. Thus, generations of stars differ in terms of their size, chemical composition, and other characteristics. Only through the change of several generations of objects this class of objects acquires some features that, nevertheless, are considered to be typical for the whole class of objects (thus, biological species are defined when, in nature, it is not possible for matings between individuals from each species to produce fertile offspring. However, many species reproduce asexually).

\subsection{Individuality as a way to increase evolutionary diversity}

- Individual fates within evolution. It appears possible to maintain that with the formation of stars one observes the emergence of individual objects in nature, 'individuals' that, on the one hand, are rather similar, but have rather different individual fates much depending on circumstances of their birth and various contin gencies. For example, stars with small mass-

see Grinin, Markov, and Korotayev 2008; Grinin 2017.

11 For example, the Solar System emerged from the remnants of a supernova explosion. It is believed that due to this fact there are so many heavy and super-heavy elements on the Earth and other planets. es (in which nuclear fusion occurs at a slow rate) can use all of their fuel (i.e., remain in the main sequence) for many billions of years. On the other hand, blue giants (in which the rate of fuel consumption is rapid and which lose most part of their mass due to their instability) burn out hundreds of times faster.

The stars can end their lives in a rather different way. Some of them, having lost one or a few outer layers, would cool, slowly transforming into cold bodies; some others may contract a few dozen times, or may end their lives with huge explosions blowing their matter into open space. Finally, a star may become a black hole that does not allow any matter to come out of its immensely compressed depths.

- Ontogenesis and phylogenesis. The evolution proceeds at various levels: through the development of its certain branch, a certain class, species and so on (and sometimes even at the level of an individual organism). In addition, applying biological terminology, at every level of evolution we find a combination of processes of ontogenesis and phylogenesis. Of course, within star-galaxy evolution the phylogenesis is represented much weaker than in the evolution of life. Nevertheless, it still appears possible to speak about the history of transformation of certain types of galaxies and stars, and, hence, up to a certain extent the cosmic phylogenesis does occur (see as above with respect to change of a few generations of stars and galaxies that differ from each other as regards their size, structure, and composition).

- Phases of individual development (ontogenesis) generate trillions of different destinies. Every type of objects has their own regular phases of life which depend on both internal characteristics of an object and its environment (proximity of other objects, etc.). As we have already pointed above, stars have very different duration of the phase which is called the main sequence (from several tens of millions years to $10-15$ billion of years and even more) depending on their mass, composition and 
other characteristics. As was mentioned above, the fate of stars at the last stage of their life also depends on their mass and other circumstances. Depending on this they can turn into the White Dwarf, become a neutron star or a Black Hole.

- Required and excessive variation as conditions of a search for new evolutionary trajectories. Within the processes described above one can observe the formation of the taxonomic diversity of space objects; we may even speak about occupying the evolutionary 'niches'. There emerge different types of stars with different masses, luminosity (accordingly, different spectrum/color of the light), temperature, system (single stars, planet systems and systems of stars from two to seven), period of rotation, magnetic field, etc. The same refers to the galaxies among which one can distinguish a number of types (elliptical, spiral, and lenticular) and subtypes. Such diversity is extremely important. Only the achievement of a necessary level of taxonomic and other diversity allows a search for ways to new evolutionary levels. This is sometimes denoted as the rule of necessary and excessive diversity (see Grinin, Markov, and Korotayev 2008: 68-72; for more details see also Panov 2008).

- Norm, averages, and deviation from a norm. Only when we find a sufficient diversity, it appears possible to speak about norm, average level, exceptions, and outliers. Scientists have long known that the breakthroughs to new forms usually happen at the periphery, and in those systems that diverge from the previous mainstream.

- Continuity, which actually means the emergence of a continuum of forms, sizes, life spans, and lifecycles, is rather characteristic for space objects. Thus, the stars can be presented as a continuum from heavier to lighter ones (whereas the latter become hardly distinguishable from planets, their temperature prevent thermonuclear reactions, etc. The types of planetary systems uniformly cover a wide range of parameters. There is also a sequence of phases in the transformation of cosmic clouds into stars: condensation of clouds - formation of protostars - formation of young stars, and up to the death of stars. The continuum of forms and sizes of objects may be observed at geological, biological, and social phases of the evolution.

\subsection{Object, environment, competition, develop- ment systems, and self-preservation}

- The relations between structure and environment. Multilevel systems (galaxy - galaxy cluster - galaxy supercluster) act as systems of a higher order for stars, and, simultaneously, they create an environment that produces an enormous influence on those stars. A star directly interacts with its immediate environment (e.g., with neighboring stars because of the strong gravity which affects the movement of both stars), whereas with the distant environment the interaction proceeds at higher levels. Within star-galaxy evolution the role of environment is generally less important than at other evolutionary levels, because single stars are separated by great distances and that is why collide rather infrequently. On the other hand, one should not underestimate the role of the environment. For example, the role of the immediate environment is very important in systems of double, triple, or multiple stars. On the whole, single stars are separated by great distances and that is why they collide rather infrequently except for the center of the galaxies where star density is much higher. Here the frequency of collisions is one per a million years (Shklovskii 1978: Ch. 1). For a small galaxy the influence of neighboring larger galaxy may turn out to be fatal, if it leads to its absorption. External factors play the major role in changes (e.g., a large cosmic body can pass by a giant molecular clouds, there can occur 
a star explosion, and so on) and may trigger the process of formation of stars and galaxies (by launching the gas contraction process). Collisions of cosmic bodies may create new cosmic bodies - for example, there is a hypothesis that the Moon emerged as a result of the collision of some large objects with the Earth.

With the development of a certain form of evolution, its own laws and environment gain a growing influence on the development of its objects and subjects. For example, both abiotic nature and the biotic environment influence biological organisms. However, within a complex ecological environment, it is the intraspecies and interspecies competition that may have larger influence than any other natural factors, whereas within a complex social environment it is just the social surrounding that affects individuals and social systems more than the natural forces do. Thus, with the formation of star-galaxy structure of the Universe there emerged macro-objects which start to interact with environments which are larger by many orders of magnitude.

- The formation of evolutionary driving forces of development. The study of cosmic evolution shows that evolutionary driving forces emerge just at this phase of evolution (although they turn to have small-scale impact on 'progress'). Of course, evolutionary changes are determined by the influence of physical or chemical forces, but we observe them sometimes in the form of preadaptations. For example, the emergence of organic chemical compounds in the clouds of molecular gas exemplifies such a preadaptation. In principle such kinds of complex compounds do not play a significant role in cosmic evolution, but they are 'in support' of development. It is of interest that it is just a peculiar structure of such clouds that protect molecules from cosmic radiation thus, making their existence possible. In other words, preadaptations require specific conditions. In biology preadaptations often emerge in peculiar environment. Thus, it is supposed that at the origin of the amphibians the crossopterygian fins (which already in many ways resembled the amphibian limbs) were transformed into primitive limbs (serving to support body on a solid ground) in the situation of shrinking shallow water.

- The urge toward self-preservation and origins of the struggle for resources. Stars, galaxies, and planets (as well as other cosmic bodies) have their definite, quite structured, and preserved form. The 'struggle' for the preservation of those forms, the capacity to live and shine, the use of different layers to minimize energy losses lead to a slow but evident evolutionary development. This way the atomic composition of the Universe changes, whereas the diversity of variations of the existence of matter increases. The bilateral transition of matter into atomic (in hot bodies) or molecular state (in cold structures, in particular in the clouds of gas and in stellar outer layers) and vice versa when forming stars from giant clouds is the brightest manifestation of this type of evolution, a background preparing the formation of its biochemical and biological forms.

On the one hand, the emergence of structures that strive for their preservation creates a wide range of interaction between the system and its environment; on the other hand, this creates a basis for the 'evolutionary search' and evolutionary advancement. This evolutionary paradox - the struggle for the self-preservation is the most important source for development - can be observed here in its full-fledged form. However, star-galaxy evolution demonstrates the emergence of this driving force which will become very important in biological evolution; and it appears to be the most important driving force in social evolution. This is the struggle for resources that among stars and galaxies may proceed in the form of weakening of another object or its destruction (e.g., through a 
direct transfer of energy and matter from one body to another), in the form of 'incorporation', 'capturing', that is 'annexation' of stars and star clusters by larger groups. We have already mentioned above galactic coalescences. Thus, some astronomers maintain that throughout a few billions of years our galaxy has 'conquered, robbed, and submitted' hundreds of small galaxies, as there are some evident 'immigrants' within our galaxy, including the second brightest star in the northern sky, Arcturus (Gibson and Ibata 2007: 30). It is widely accepted that emergence and expansion of a black hole may lead to the 'eating' of the matter of the nearby stars and galaxies. However, the 'eating capacity' of the black holes is greatly exaggerated in popular literature. In systems of double stars or in starplanet systems one may also observe such a form of interaction as the exchange of energy and resources.

- External factors as triggers of transformations play a great role, for example, when a large celestial object passes close to giant molecular clouds or a star explodes, etc. this can launch the process of stars and galaxies formation (i.e. trigger gas concentration). The collisions between celestial bodies can form new objects. Thus, it is supposed that the Moon emerged as a result of a collision of a large object with the Earth. The shockwave from a nearby supernova may have been such a trigger for the birth of the Sun about two million years before its collapse started (see Adushkin et al. 2008, 276; Bizzarro et al. 2007; Grinin 2017, 2018).

Thus, we can formulate rule of necessary triggering phenomena or events to launch evolutionary process. A push or trigger is often needed to launch the phase transition or transformation of an object. On the one hand, the latter will not work without internal readiness of a system, but on the other - even a high level of internal readiness cannot start up the transformation process itself, like gunpowder cannot explode without fire. Without a trigger, the system can remain in the state of potential readiness for transformations for a long time. In this case, analogues of evolutionary typical / recognized systems emerge.

\subsection{Multilinearity}

Multilinearity is one of the most important characteristics of evolution. Unfortunately, it does not get sufficient attention, and there is a tendency to reduce evolution to a single line - the one that has produced the highest complexity level, which is often interpreted as the main line of evolution. However, at every stage of evolutionary development one can find an interaction of a few lines that can have rather different futures. In other words, in addition to the main evolutionary line one can always identify a number of lateral ones. Firstly, they contribute to the increasing diversity; secondly, they allow expanding the range of search opportunities to move to new levels of development; thirdly, the lateral lines may partly enter the main evolutionary stream, enriching it. We quite often deal with two or more coexisting and comparable lines of development whose convergence may lead to a quantitative breakthrough and synergetic effect. Various lines of development may transform into each other. Elsewhere we have written a lot on the issue of social evolution in this context (see, e.g., Grinin and Korotayev 2009; Grinin and Korotayev 2011; Bondarenko, Grinin, and Korotayev 2011; Grinin 2011).

- Classical forms and their analogues. The main and lateral lines of evolution may be considered in two dimensions: 1) horizontal (as regards complexity and functions), 2) vertical (concerning the version that would be realized later at higher evolutionary phases). It appears also possible to speak about classical versions and their analogues. Thus, various forms of aggregation and specialization of unicellulars can be regarded as analogues of multicellulars (see Eskov 2006), whereas various complex stateless polities can be regarded as state analogues (see Grinin and Korotayev 2006; Grinin and Korotayev 2009; Grinin 2011 for more detail). Classical forms and their analogues can transform into each other; however, these are just the analogues that tend 
to transform into classical forms, rather than the other way round (the latter may be regarded as a forced adaptation to sharply changing conditions, and sometimes even as a direct degeneration).

- Stars and molecular clouds: two parallel forms of existence of cosmic matter. In this respect we may consider stars and galaxies as the main line of evolution and the giant clouds as its lateral lines; the former may be denoted as 'classical forms', and the latter may be designated as 'analogues'. On the one hand, those forms actually transform into each other. Galaxies and stars emerge from giant molecular clouds, whereas stars through explosions and shedding their envelopes may transform into gas-dust cloud. On the other hand, giant molecular clouds are able to concentrate; the energy exchange occurs within them, and thus, in terms of gravity and structural complexity they are quite comparable to stars and galaxies ${ }^{12}$. They also exceed stars in the level of organization of elementary particles since molecules are concentrated in clouds, while elementary particles and nuclei - in stars. ${ }^{13}$ Besides, stars when they lose the matter and shed their envelopes and due to an explosion transit into gas-dust state, i.e. into interstellar gas which gathers into molecular clouds.

\section{Conclusion: The Formationof Various}

\section{Evolutionary Lines at the Microworld Level}

Astrophysical and astrochemical evolution. Almost from the very beginning of the development of the Universe (when the temperature reached thousands of Kelvin) chemical evolution emerges as accompanying physical and astrophysical evolution. Of course, chemical evolution also occurs within stars with the emergence of heavier elements. However, that was rather the formation of the basis for chemical evolution, because chemical processes involve

12 At various levels of generalization, the clouds of the same size are considered the analogues of stars, while of the larger ones - of galaxies.

13 The molecules can also be found in periphery layers of some stars, in the areas with low temperatures. the reactions which lead to the emergence of new substances. Such processes proceed, first of all, within gas-dust clouds where molecules emerge. Hydrogen molecules are absolutely prevalent quantitatively; however, molecules of water and some other substances also emerged. Chemical evolution goes on also on planets (where it combines with geological, or rather planetary evolution) as well as on small celestial bodies (asteroids and meteorites). At the same time, on the planets where due to volcanism, pressure and other geological processes the temperatures could be high enough, the chemical activity significantly differed from that in cold clouds.

Chemical evolution within cosmic evolution. Following Friedrich Engels (and his 'Dialectics of Nature' [Engels 1940]), the representatives of dialectic materialism stated that the chemical form of organization of matter is evolutionarily higher than the physical one. However, in contrast with biological and social forms which from their very origin displayed substantially higher levels of organization of the matter, the chemical form (that emerged almost immediately after the physical form) did not represent a higher form of evolution for a rather long period of time. This is not the case of geological (or rather planetological) evolution which emerged on the planets long time ago and advanced evolution (including the chemical one) through the formation of various minerals and of more comfortable temperature conditions for the development, as well as formation of atmospheres and even of hydrosphere in some places (like on Mars). However, the further evolutionary progress became possible only as a preparation for the formation of appropriate conditions for life on Earth.

That is not to say that chemical evolution is not important in the framework of general stellar and galactic evolution; however, before the emergence of the Earth-like planet, the physical and chemical forms of organization of matter should be regarded as equally important; note also that they constantly transform into each other (see also Dobrotin 1983: 89) ${ }^{14}$. The

14 In any case, it is important to note that the thermonuclear 
development of astrochemical evolution is not limited by the formation of simple nonorganic molecules. The processes of formation of molecules proceed further towards the formation of organic substances. More than hundred types of organic molecules have been detected in space (including 9-13 atomic molecules) even including ethanol and amino acid Glycine (see Surdin 2001; Surdin and Lamzin 1992; Shklovskii 1978). Naturally, this facilitated the emergence of life in a rather significant way.

The preadaptation is also manifested in the chemical reactions of a peculiar type which proceed in gas-dust clouds 'not in the usual way, but through a quantummechanical subbarrier transition, which does not require much kinetic energy from the participants of the reaction' (Surdin, Lamzin 1992 ). In other words, multilinearity is also expressed in the fact that classical chemical reactions (which could already take place on some planets) have their analogues. As a result, the multilinearity of evolution is further realized in the set of achievements of its various lines (chemical and geological ones), as it happened on Earth, which gave it an opportunity to move to a new evolutionary level.

\section{The Formation of 'Preadaptations' as Points of Fu- ture Evolutionary Growth}

Within the star-galaxy era the chemical form of development may be regarded as a 'preadaptation' for new levels of evolution. Let us note that in biology the term 'preadaptation' denotes those adaptations that may turn out to be useful in a different environment and to give significant advantages to those species that have them ${ }^{15}$ - and generally - to give an impulse to the formation of new taxa.

Within the Big History framework, the principle of 'preadaptation' means that at the level where a

reactions make the chemical evolution of the Galaxy proceed in a single direction, namely, from simple to complex elements (Surdin and Lamzin 1992). This also refers to evolution in general.

15 This omnivorous ability of hominids allowed their transition to hunting at a very early phase of the anthropogenesis. preadaptation emerges, it generally plays insignificant role; however, at a new evolutionary level such 'innovations' generally give evolutionary impulses. ${ }^{16}$ Respectively, chemical compounds (as is common for preadaptations) do not mean much for cosmic evolution, they were rather 'in reserve' to reveal all their significance at the level of planetary evolution.

I would like to finish this article with a note on one more peculiarity of preadaptations. Appropriate conditions are necessary for their formation. Within biological evolution, the preadaptations often emerge in peculiar environments. Thus, it is supposed that the transformation of fins of the fleshy-finned fish (from which Amphibia descended) into primitive legs occurred within the environment of shallow waters that often dried out. In a similar way, within star-galaxy evolution the emergence of complex chemical compounds can take place only within certain structures of cosmic clouds that made their existence possible as they protected the molecules from cosmic radiation.

\section{Conclusion}

So it is important to summarize our approach which, in our opinion, is fruitful in the field of Big History, as in any other researches about evolutionary analogues between cosmology, society and biological world.

Wecan see differentmechanisms, such as: catastrophes, self-preservation, interaction with environment, struggle for resources, formation of individual objects, formation of the main and lateral lines, diversity, selection, etc., which demonstrate the commonality of evolutionary rules and patterns in different phase of Big History and different levels of evolution. Unfortunately we can't pay enough attention to each mechanism and rule, because for a detailed explanation it would be necessary to write a special article.

It would be worth to mention that some authors regard natural selection as the main and sometimes the only one evolutionary mechanism, which works not only in biological stage of Big History, but in

16 On preadaptations in megaevolution see also Grinin, Korotayev, and Markov 2011: 159-160. 
cosmological stage too. Without any doubt selection is important and along with other evolutionary mechanisms can provide insights of the formation of the large-scale structure of the Universe. However, as the reader can understand, we regard the selection mechanism only as one of the most important for cosmology, but by no means the main one, and, moreover, not the only one.

Nevertheless, one cannot but give credit to many interesting cosmological hypotheses. For example, David Baker explores the selection mechanism among an enormous number of universes in the 'multiverse'. However, his algorithm with respect to the selection of universes could hardly be called properly Darwinian. He rather speaks about the evolutionary selection in general - that is not the selection of the fittest, but rather the selection of those capable to evolve - which is much wider than the Darwinian selection. The idea that such selection is not Darwinian is confirmed if one employs Christian's (2014) and Smolin's (2008) works ${ }^{17}$.

In conclusion, we want to repeat the objectives of the article. First of all, we used a historical narrative showing the origin and development of general evolutionary models and then explaining them to give an idea of the formation of the large-scale structure of the Universe.

Secondly, the goal was to find evolutionary similarities at different levels and to use them for the analysis of commonality of evolutionary rules and patterns in different systems.

We did not try just to apply already formulated evolutionary principles of different complex biological or social systems to the cosmological issues. This method can be wrong, since many of such principles will be unsuitable in cosmology.

Thirdly, but, rather briefly, some questions were discussed about analogues of functioning and developing between various objects and systems.

\section{References}

\section{Adushkin,V. V., Vityazev, A. V., and Pechernikova}

17 Smolin often refers to the anthropic principle as a very important evolutionary idea, which we did not touch in the article.
G. 2008. To the Elaboration of the Theory of the Origin and Early Evolution of the Earth. In Eduard Galimov (ed.), Issues of the Origin and Evolution of the Biosphere (pp. 275-296). Moscow: LIBROKOM. In Russian (Адушкин, В. В., Витязев, А. В., Печерникова, Г. В. В развитие теории происхождения и ранней эволюции Земли. Проблемы зарождения и эволюции биосферы: сб. науч. работ / под ред. Э. М. Галимова, с. 275 296. М.: ЛИБРОКОМ).

Baade, W. 2002. Evolution of Stars and Galaxies. Moscow: URSS. In Russian (Бааде В. Эволюция звезд и галактик. М.: УРСС).

Baker, D. 2013. $10^{500}$. The Darwinian Algorithm and a Possible Candidate for a 'Unifying Theme' of Big History. In Grinin, L. E., and Korotayev, A. V. (eds.), Evolution: Development within Big History, Evolutionary and World-System Paradigms. Yearbook (pp. 235-248). Volgograd: Uchitel.

Batygin, K., Laughlin G., and Morbidelli, A. 2016. Born of Chaos. Scientific American 5 (314): 28-37.

Bizzarro, M. et al. 2007. Evidence for a Late Supernova Injection of $60 \mathrm{Fe}$ into the Protoplanetary Disk. Science 316 (5828): 1178-1181. Doi:10.1126/science. 1141040.

Bondarenko, D. M., Grinin, L. E., and Korotayev, A. V. 2011. Social Evolution: Alternatives and Variations (Introduction). In Grinin, L. E., Carneiro, R. L., Korotayev, A. V., and Spier F. (eds.), Evolution: Cosmic, Biological, and Social (pp. 212-250). Volgograd: Uchitel.

Christian D. 2004. Maps of Time: an Introduction to Big History. Berkeley, CA: University of California Press.

Christian D. 2014. Swimming Upstream: Universal Darwinism and Human History. In Grinin, L. E., Baker, D., Quaedackers, E., and Korotayev, A. V. (eds.), Teaching \& Researching Big History: Exploring a New Scholarly Field (pp. 19-40). Volgograd: 'Uchitel' Publishing House. 
Diemand, J., Kuhlen, M., Madau, P., Zemp, M., Moore, B., Potter, D., and Stadel, J. 2008. Clumps and Streams in the Local Dark Matter Distribution. Nature 454(7205): 735-738.

Dolgov, A. D., Zeldovich, Ya. B., and Sazhin, M. V. 1998. Cosmology of the Early Universe. Moscow: Moscow University Press. In Russian (Долгов A. Д., Зельдович Я. Б., Сажин М. В. Космология ранней Вселенной. M: Издательство Московского Университета).

Efremov, Yu. N. 2003. Deep into the Universe. Moscow: URSS. In Russian (Ефремов Ю. Н. Вглубь Вселенной. М.: Едиториал УРСС).

Eskov, K. Yu. 2006. Amazing Paleontology. History of the Earth and Life on It. Moscow: Enas. In Russian (Еськов К. Ю. Удивительная палеонтология. История Земли и жизни на ней. М: Энас).

European Commission - MEMO 2011. EU Marie Curie researcher discovers galaxy 13 billion light years away. URL: http://europa.eu/rapid/pressrelease_MEMO-11-237_en.htm.

Genet, R. M. 2007. Humanity: The Chimpanzees Who would be Ants. Santa Margarita, CA: Collins Foundation Press.

Gibson, B., and Ibata, R. 2007. The Phantom of Dead Galaxies. $V$ mire nauki, June: 29-35. In Russian (Гибсон Б., Ибата Р. Призраки погибших галактик. В мире науки, июнь: 29$35)$.

Gorbunov, D. S., and Rubakov, V. A. 2011. Introduction to the Theory of the Early Universe: Cosmological Perturbations and Inflationary Theory. Singapore - Hackensack, NJ: World Scientific Publishing Company.

Gorbunov, D. S., and Rubakov, S. A. 2012. Introduction to the Theory of the Early Universe. The Theory of Big Bang. Moscow: LKI. In Russian (Горбунов Д. С., Рубаков С. А. Введение в теорию ранней Вселенной. Теория горячего Большого взрыва. 2-е изд. М.: ЛКИ).

Grinin, A. L. 2016. 'elf-regulation as a global evo- lutionary mega-trend. In Grinin, L.E., and Korotayev, A.V. (eds) Evolution: Evolution and Big History: Dimensions, Trends, and Forecasts (pp. 139-64). Volgograd: Uchitel.

Grinin, L. E. 2011. The Evolution of Statehood. From Early State to Global Society. Saarbrücken: Lambert Academic Publishing.

Grinin L. E. 2013. Big History of the World Development: Cosmic Evolution. Volgograd: Uchitel. In Russian (Гринин Л. Е. 2013. Большая история развития мира: Космическая эволюция. Волгоград: Учитель).

Grinin L. E. 2014a. The Star-Galaxy Era of Big History in the Light of Universal Evolutionary Principles. In Grinin, L. E., Baker, D., Quaedackers, E., and Korotayev, A. V. (eds.), Teaching \& Researching Big History: Exploring a New Scholarly Field (pp. 163-187). Volgograd: 'Uchitel' Publishing House.

Grinin L. E. 2014b. The Star-Galaxy Era of Big History in the Light of Universal Evolutionary Principles. Evolution: From Proto-stars to Singularity? / Edited by L. E. Grinin, Andrey V. Korotayev and A. V. Markov. Volgograd: Uchitel. Pp. 78-106. In Russian (Гринин Л. Е. Звездно-галактическая эра Большой истории в свете универсальных принципов эволюции / Эволюция: от протозвезд к сингулярности? // Отв. ред. Л. Е. Гринин, А. В. Коротаев, А. В. Марков. - Волгоград: Учитель. C. 78-106).

Grinin L. E. 2015a. The Star-Galaxy Era of Big History in the Light of Universal Evolutionary Principle. Globalistics and Globalization Studies: Big History \& Global History. Yearbook / Edited by Leonid E. Grinin, Ilya V. Ilyin, Peter Herrmann, and Andrey V. Korotayev. - Volgograd: 'Uchitel' Publishing House. P. 282-300.

Grinin L. E. 2015b. Cosmic Evolution and Universal Evolutionary Principles. Evolution: From Big Bang to Nanorobots / Edited by Leonid E. Grinin and Andrey V. Korotayev. - Volgograd: 'Uchitel' Publishing House Pp. 20-45. 
Grinin, L. 2017. The Development of the World in Terms of Big History: The History and Evolution of the Solar System. Volgograd: Uchitel. In Russian (Гринин, Л. Е. Большая история развития мира: история и эволюция Солнечной системы. Волгоград: Учитель).

Grinin L. E. 2018. Evolution of the Early Solar System in Terms of Big History and Universal Evolution. Journal of Big History II(1): 15-26. http:// dx.doi.org/10.22339/jbh.v2i1.2252

Grinin, L. 2019. Was There a Big Bang? Journal of Big History III(1): 51-59.

Grinin, L. E., and Korotayev, A. V. 2006. Political Development of the World System: A Formal Quantitative Analysis. In Turchin, P., Grinin, L., Korotayev, A., and de Munck, V. (eds.), History and Mathematics. Historical Dynamics and Development of Complex Societies (pp. 115-153). Moscow: KomKniga/URSS.

Grinin, L. E., and Korotayev, A. V. 2009. The Epoch of the Initial Politogenesis. Social Evolution and History 8(1): 52-91.

Grinin, L. E., and Korotayev, A. V. 2011. Chiefdoms and Their Analogues: Alternatives of Social Evolution at the Societal Level of Medium Cultural Complexity. Social Evolution and History. Special Issue. Chiefdoms: Theories, Problems, and Comparisons 10(1): 276-335.

Grinin, L. E., Korotayev, A. V., and Rodrigue, B. H. 2011. Introduction. Evolution and Big History: From Multiverse to Galactic Civilizations. In Grinin, L. E., Korotayev, A. V., and Rodrigue, B. H. (eds.), Evolution: A Big History Perspective (pp. 5-19). Volgograd: Uchitel.

Grinin, L. E., Markov, A. V., and Korotayev, A. V. 2008. Macroevolution in Wild-life and Society. Moscow: LKI. In Russian (Гринин, Л. Е., Марков, A. В., Коротаев, A. В. Макроэволюция в живой природе и обществе. М.: Издательство ЛКИ).

Guth, A. H. 1997. Was Cosmic Inflation the 'Bang' of the Big Bang? Beem Line 27 (3). URL: http://ned.
ipac.caltech.edu/level5/Guth/Guth1.html

Guth, A. 2002. The Inflationary Universe. URL: http://www.edge.org/conversation/the-inflationary-universe-alan-guth

Guth, A. 2004. Inflation. In Freedman, W. L. (ed), Carnegie Observatories Astrophysics Series. Vol. 2: Measuring and Modeling the Universe. Cambridge: Cambridge University Press. URL: http:// www.astro.caltech.edu/ george/ay21/ readings/ guth.Pdf

Hawking, S. 1998. A Brief History of Time. From the Big Bang to Black Holes. New York: Bantam Books.

Khvan, M. P. 2008. Furious Universe. From Big Bang to Accelerating Expansion, from Quarks to Superstrings. Moscow: LKI. In Russian (Хван M. П. Неистовая вселенная. От Большого взрыва до ускоренного расширения, от кварков до суперструн. М.: ЛКИ).

Lin, Douglas N. C. 2008. The Genesis of Planets. Scientific American 298(5): 50-59. doi:10.1038/scientificamerican0508-50.

Lipunov, V. M. 2008. In the World of Binary Stars. Moscow: LIBROKOM. In Russian (Липунов B. М. В мире двойных звезд. М.: ЛИБРОКОМ).

Marakushev, A. A. et al. 2013. Origin and Evolution of the Solar System. Prostranstvo i vremya 2 (12): 132-141. In Russian (Маракушев, А. А., Зиновьева, Н. Г., Панеях, Н. А., Маракушев, С. А. Зарождение и эволюция Солнечной системы. Пространство и Время. Вып. 2 (12): 132-41).

Marov, M. et al. 2008. From a Proto-Solar Nebula to a Planetary Systam: The Model of the Emergence of the Gas-Dust Disk. In Galimov, E. M. (ed.), Issues of the Origin and Evolution of the Biosphere (pp. 223-274). Moscow: Librokom. In Russian (Маров, М. Я., Колесниченко, А. В., Макалкин, А. Б., Дорофеева, В. А., Зиглина, И. Н., Чернов, А. В. От протосолнечного облака к планетной системе: Модель эволюции газопылевого диска. Проблемы зарождения и эволюции биосферы: 
сб. науч. работ / под ред. Э. М. Галимова, с. 223-74. М.: ЛИБРОКОМ).

May, B., Moore, P., and Lintott, C. 2008. Bang: The Complete History of the Universe. Baltimore, MD: Johns Hopkins University Press.

Novikov I. D. 1979. Evolution of the Universe. Moscow: Nauka. In Russian (Новиков И. Д. Эволюция Вселенной. М.: Наука).

Panov, A. D. 2008. Cohesion of Social and Biological Evolution and the Limit of Its Acceleration. Historical Psychology and Sociology of History 2: 25-48. In Russian (Панов А. Д. Единство социально-биологической эволюции и предел ее ускорения. Историческая психология и социология истории 2: 25-48).

Pavlov, A. N. 2011. Geophysics. The General Course on the Nature of the Earth. St. Petersburg: Russian State Hydrometeorological University. In Russian (Павлов А. Н. Гео-физика. Общий курс о природе Земли. СПб.: РГГМУ).

Peebles P. J. E. 1980. The Large-Scale Structure of the Universe. Princeton: Princeton University Press.

Ryabov, V. A., Tsarev, V. A., Tskhovrebov, A. M. 2008. Search for Dark Matter. Uspekhi fizicheskikh nauk 178(11): 1130-1164. In Russian (Рябов B. А., Царев В. А., Цховребов А. М. 2008. Поиски частиц темной материи. Успехи физических наук 178(11): 1130-1164).

Schumpeter, J. A. 1994 [1942]. Capitalism, Socialism and Democracy. London: Routledge.

Shklovsky, I. S. 1984. Stars: Their Birth, Life and Death. $3^{\text {rd }}$ ed. Moscow: Nauka. In Russian (Шкловский И. С. Звезды: их рождение, жизнь и смерть. Изд. 3-е. М.: Наука).

Shklovskii, I. S. 1978. Stars: Their Birth, Life, and Death. R.B. Rodman (Translator). San. Francisco: W.H. Freeman.

Smolin, Lee. 1999. The life of the cosmos. Oxford University Press.
Spencer H. 1862. First Principles. $1^{\text {st }}$ ed. London: Williams \& Norgate.

Spencer, H. 1870. “First Principles,'Williams \& Norgate: London, Second edition.

Spier, F. 2010. Big History and the Future of Humanity. Oxford: John Wiley \& Sons.

Surdin, V. G. 2001. Stars Production. Moscow: URSS. In Russian (Сурдин В. Г. Рождение звезд. $M .:$ УPCC).

Surdin, V. G., and Lamzin, S. A. 1992. Protostars. Where, How and Out of What Stars Form. Moscow: Nauka. In Russian (Сурдин В. Г., Ламзин С. А. Протозвезды. Где, как и из чего формируются звездыл. М.: Наука).

Surkova, L. P. 2005. Stars and Stellar Clusters in Our Galaxy. Chita: Zabaikalsky State University. In Russian (Суркова Л. П. Звезды и звездные группировки в нашей Галактике. Чита: ЗабГПУ).

Teilhard de Chardin, P. 1987. The Phenomenon of Man. New York: Harper Perennial Modern Classics.

Vilenkin, A. 2006. Many Worlds in One: The Search for Other Universes. New York: Nill and Wangs.

Vilenkin, A. 2010. The World of Many Worlds. Physicists in Search for Other Universes. Moscow: Astrel. In Russian (Виленкин A. Мир многих миров. Физики в поисках иных вселенных. М.: Астрель).

Weinberg S. 1977. The First Three Minutes: A Modern View of the Origin of the Universe. Updated Edition. New York: Basic Books.

Wood, B. 2018. Imagining the Unimaginable: Narratives of the Big Bang Time, Space, Matter, Energy. Journal of Big History 2(1): 1-13. URL: http://dx. doi.org/10.22339/jbh.v2i1.2251. 